\title{
Further Approximations for Elliptic Integrals
}

\author{
By Yudell L. Luke*
}

\begin{abstract}
The present paper develops approximations for the three kinds of elliptic integrals based on the Padé approximations for the square root. The work includes and extends our previous work on the subject to provide efficient approximations over a larger part of the complex $k$ and $\phi$ planes.
\end{abstract}

1. Introduction. In previous work [1], [2], we gave some approximations for the three kinds of complete and incomplete elliptic integrals which were derived by using Padé approximations for the square root. The expansions are valid in a large sector of the complex plane and are far more powerful than the analogous representations based on the binomial expansion for the square root. Nonetheless, the convergence properties of our previous developments weaken for $k^{2} \sin ^{2} \phi$ near unity. In this paper, we develop new representations like those of [1], [2] to correct this deficiency. The present study is suggested by analogous work for power series developments of the first two kinds of elliptic integrals. In this connection, see the recent study by Van de Vel [3] and the references quoted there. For convenience and completeness, we also give the representations previously found. Our proofs are very sketchy as the details are akin to those previously used.

\section{Approximations for the Square Root and Other Data.}

$$
\begin{aligned}
(1-z)^{-1 / 2} & =(2 n+1)^{-1} \sum_{m=0}^{n} \epsilon_{m}\left(1-z \sin ^{2} \theta_{m}\right)^{-1}+V_{n}(z), \\
(1-z)^{1 / 2} & =1-2 z(2 n+1)^{-1} \sum_{m=1}^{n} \frac{\sin ^{2} \theta_{m}}{1-z \cos ^{2} \theta_{n}}+W_{n}(z), \\
\epsilon_{m} & =1 \quad \text { if } m=0, \quad \epsilon_{m}=2 \text { if } m \geqq 1, \\
\theta_{m} & =m \pi /(2 n+1) .
\end{aligned}
$$

Let

$$
e^{\zeta}=\frac{2-z \pm 2(1-z)^{1 / 2}}{z}
$$

where the sign is chosen so that $\left|e^{\xi}\right|$ lies outside the unit disc which is possible for all $z$ except $z \geqq 1$. Then

$$
V_{n}(z)=\frac{4 e^{-(2 n+3 / 2) \zeta}}{z^{1 / 2}\left(1-e^{-\zeta}\right)\left[1+e^{-(2 n+1) \zeta}\right]},
$$

Received July 30, 1969.

AMS Subject Classifications. Primary 33.19, 41.17.

Key Words and Phrases. Elliptic integrals, approximations by rational functions.

* This research was supported by the U. S. Atomic Energy Commission under Contract No. AT(11-1) 1619 . 


$$
W_{n}(z)=-\frac{z^{1 / 2}\left(1-e^{-\zeta}\right) e^{-(2 n+1 / 2) \zeta}}{\left[1-e^{-(2 n+1) \zeta}\right]}
$$

and for $z$ fixed,

$$
\lim _{n \rightarrow \infty}\left\{V_{n}(z) \text { and } W_{n}(z)\right\}=0, \quad z \neq 1,|\arg (1-z)|<\pi .
$$

It is also convenient to have representations like (1) and (2) with $z$ replaced by $-z$. Thus

$$
\begin{aligned}
& (1+z)^{-1 / 2}=(2 n+1)^{-1} \sum_{m=0}^{n} \epsilon_{m}\left(1+z \sin ^{2} \theta_{m}\right)^{-1}+R_{n}(z), \\
& (1+z)^{1 / 2}=1+2 z(2 n+1)^{-1} \sum_{m=1}^{n} \frac{\sin ^{2} \theta_{m}}{1+z \cos ^{2} \theta_{m}}+S_{n}(z) .
\end{aligned}
$$

For the error terms, let

$$
e^{\eta}=\frac{2+z \pm 2(1+z)^{1 / 2}}{z}
$$

where the sign is chosen so that $\left|e^{\eta}\right|$ lies outside the unit disc, which is possible for all $z$ except $-1 \geqq z \geqq-\infty$. Then

$$
\begin{aligned}
& R_{n}(z)=-\frac{4 e^{-(2 n+3 / 2) \eta}}{z^{1 / 2}\left(1+e^{-\eta}\right)\left[1-e^{-(2 n+1) \eta}\right]}, \\
& S_{n}(z)=\frac{z^{1 / 2}\left(1+e^{-\eta}\right) e^{-(2 n+1 / 2) \eta}}{\left[1+e^{-(2 n+1) \eta}\right]},
\end{aligned}
$$

and for $z$ fixed,

$$
\lim _{n \rightarrow \infty}\left\{R_{n}(z) \text { and } S_{n}(z)\right\}=0, \quad z \neq-1,|\arg (1+z)|<\pi .
$$

Finally, we have need for some integrals:

$$
H\left(\theta, a^{2}\right)=\int_{0}^{\theta} \frac{d \alpha}{1-a^{2} \sin ^{2} \alpha} .
$$

$$
\begin{gathered}
H\left(\theta, a^{2}\right)=c^{-1} \arctan (c \tan \theta), \quad c=\left(1-a^{2}\right)^{1 / 2} \\
\left|\arg \left(1-a^{2}\right)\right|<\pi, \quad|\arg (1 \pm i c \tan \theta)|<\pi, \quad i=(-1)^{1 / 2} \\
H\left(\theta, a^{2}\right)=(2 g)^{-1} \ln \left|\frac{1+g \tan \theta}{1-g \tan \theta}\right|, g=\left(a^{2}-1\right)^{1 / 2}, g \tan \theta \text { real } \\
H\left(\theta, a^{2}\right)=\tan \theta \text { if } a^{2}=1 \\
=\pi / 2 c \text { if } \theta=\pi / 2 \text { and }\left|\arg \left(1-a^{2}\right)\right|<\pi \\
=0 \text { if } \theta=\pi / 2 \text { and } a^{2}>1 . \\
\int_{0}^{\theta} \frac{\sin ^{2} \alpha}{1-a^{2} \sin ^{2} \alpha} d \alpha=a^{-2}\left[H\left(\theta, a^{2}\right)-\theta\right] .
\end{gathered}
$$

In the above, the integrals are to be interpreted in the Cauchy sense when $a^{2} \sin ^{2} \theta$ 
1. Indeed, throughout this paper, we interpret integrals in this sense when appropriate.

$$
\begin{aligned}
& \text { (20) } J\left(\theta, a^{2}\right)=\int_{0}^{\theta} \frac{\cos \alpha d \alpha}{\cos ^{2} \alpha+a^{2} \sin ^{2} \alpha}=\int_{0}^{\theta} \frac{\cos \alpha d \alpha}{1-\left(1-a^{2}\right) \sin ^{2} \alpha}, \\
& \text { (21) } J\left(\theta, a^{2}\right)=(2 c)^{-1} \ln \frac{1+c \sin \theta}{1-c \sin \theta}, \quad c=\left(1-a^{2}\right)^{1 / 2}, \quad c \sin \theta \neq 1, \\
& \text { (22) } J\left(\theta, a^{2}\right)=(2 c)^{-1} \ln \left|\frac{c \sin \theta+1}{c \sin \theta-1}\right|, \quad c \sin \theta \text { real . } \\
& \text { (23) } J\left(\theta, a^{2}\right)=\sin \theta \text { if } a^{2}=1 . \\
& \text { (24) } J\left(\theta, a^{2}\right)=g^{-1} \text { arc tan }(g \sin \theta), \quad g=\left(a^{2}-1\right)^{1 / 2}, \quad g \sin \theta \text { real. }
\end{aligned}
$$

3. Approximations for the Elliptic Integrals of the First and Third Kinds. Let

$$
\begin{aligned}
F(\phi, k, \nu)= & \int_{0}^{\phi}\left(1-\nu^{2} \sin ^{2} \alpha\right)^{-1}\left(1-k^{2} \sin ^{2} \alpha\right)^{-1 / 2} d \alpha, \\
& \nu^{2} \sin ^{2} \phi \neq 1, \quad\left|\arg \left(1-k^{2}\right)\right|<\pi, \quad\left|\arg \left(1-k^{2} \sin ^{2} \phi\right)\right|<\pi .
\end{aligned}
$$

Using (1) and (15) - (18), we get

$$
\begin{aligned}
F(\phi, k, \nu)= & \frac{H\left(\phi, \nu^{2}\right)}{2 n+1} \sum_{m=0}^{n} \epsilon_{m}\left(1-\frac{k^{2}}{\nu^{2}} \sin ^{2} \theta_{m}\right)^{-1} \\
& -\frac{2 k^{2}}{(2 n+1) \nu^{2}} \sum_{m=1}^{n} \frac{\sin ^{2} \theta_{m} H\left(\phi, k^{2} \sin ^{2} \theta_{m}\right)}{1-\left(k^{2} / \nu^{2}\right) \sin ^{2} \theta_{m}} \\
Q_{n}(\phi, k, \nu)= & \int_{0}^{\phi}\left(1-\nu^{2} \sin ^{2} \alpha\right)^{-1} V_{n}\left(k^{2} \sin ^{2} \alpha\right) d \alpha \\
Q_{n}(\phi, k, \nu)= & \frac{2 e^{-(2 n+1) \zeta} \tan \phi}{\left(1-\nu^{2} \sin ^{2} \phi\right)(4 n+3)}\left[1+\frac{\lambda_{1}}{4 n+3}+O\left(n^{-2}\right)\right]+O\left(e^{-4 n \zeta}\right), \\
\lambda_{1}= & 1-\frac{\cosh \zeta-1}{\sinh \zeta \cos ^{2} \phi}-\frac{2 \nu^{2} \sin ^{2} \phi(\cosh \zeta-1)}{\left(1-\nu^{2} \sin ^{2} \phi\right) \sinh \zeta},
\end{aligned}
$$

$$
\begin{aligned}
& \phi \neq \text { an odd multiple of } \frac{\pi}{2}, \\
& Q_{n}\left(\frac{\pi}{2}, k, \nu\right)=\left[\frac{2 \pi \sinh \zeta}{(4 n+3)(\cosh \zeta-1)}\right]^{1 / 2} \frac{e^{-(2 n+1) \zeta}}{\left(1-\nu^{2}\right)}\left[1-\frac{\lambda_{2}}{4 n+3}+O\left(n^{-2}\right)\right] \\
&+O\left(e^{-4 n \zeta}\right), \\
& \lambda_{2}= \frac{\left(2-k^{2}\right)(\cosh \zeta+1)}{8 \sinh \zeta}-\frac{1}{2}+\frac{\nu^{2}(\cosh \zeta-1)}{\left(1-\nu^{2}\right) \sinh \zeta},
\end{aligned}
$$

where $e^{\zeta}$ is defined by (5) with $z=k^{2} \sin ^{2} \phi$.

Clearly for fixed $\phi, k$ and $\nu$, under the conditions given in (25), 


$$
\lim _{n \rightarrow \infty} Q_{n}(\phi, k, \nu)=0 .
$$

In (21), the coefficient of $G\left(\phi, \nu^{2}\right)$ can be replaced by $\left(1-k^{2} / \nu^{2}\right)^{-1 / 2}-V_{n}\left(k^{2} / \nu^{2}\right)$ provided $\left|\arg \left(1-k^{2} / \nu^{2}\right)\right|<\pi$. This should be used only when the magnitude of $V_{n}\left(k^{2} / \nu^{2}\right)$ does not exceed the magnitude of $Q_{n}(\phi, k, \nu)$, which happens approximately when $\left|e^{\zeta}\right|$ with $z=k^{2} \sin ^{2} \phi$ exceeds $\left|e^{\zeta}\right|$ with $z=k^{2} / \nu^{2}$. In the case that $\phi, k$, and $\nu$ are real, this happens approximately when $1 \leqq \nu^{2} \sin ^{2} \phi$.

Some numerics illustrating these approximations and the striking realism of the error formulations are given in my earlier works. It is clear that the accuracy weakens for values of $k^{2} \sin ^{2} \phi$ near unity. To correct this deficiency, we proceed as follows. We can write

$$
\begin{aligned}
F(\phi, k, \nu) & =\int_{0}^{\phi} \frac{\sec \alpha d \alpha}{\left(1-\nu^{2} \sin ^{2} \alpha\right)\left(1+{k^{\prime}}^{2} \tan ^{2} \alpha\right)^{1 / 2}}, \\
k^{\prime 2} & =1-k^{2}, \quad\left|\arg \left(1+{k^{\prime}}^{2} \tan ^{2} \phi\right)\right|<\pi .
\end{aligned}
$$

Now use (9) and (20) - (24). Thus

$$
\begin{aligned}
F(\phi, k, \nu)= & \frac{1}{(2 n+1)\left(1-\nu^{2}\right)} \sum_{m=0}^{n} \frac{\epsilon_{m}\left(1-k^{\prime 2} \sin ^{2} \theta_{m}\right)}{1-\left(k^{\prime 2} /\left(1-\nu^{2}\right)\right) \sin ^{2} \theta_{m}} J\left(\phi, k^{\prime 2} \sin ^{2} \theta_{m}\right) \\
& -\frac{\nu^{2}}{(2 n+1)\left(1-\nu^{2}\right)} J\left(\phi, 1-\nu^{2}\right) \sum_{m=0}^{n} \frac{\epsilon_{m}}{1-\left(k^{\prime 2} /\left(1-\nu^{2}\right)\right) \sin ^{2} \theta_{m}} \\
& +S_{n}(\phi, k, \nu)
\end{aligned}
$$

$$
\begin{aligned}
S_{n}(\phi, k, \nu) & =\int_{0}^{\phi} \frac{\sec \alpha R_{n}\left(k^{\prime 2} \tan ^{2} \alpha\right) d \alpha}{1-\nu^{2} \sin ^{2} \alpha} \\
S_{n}(\phi, k, \nu) & =-\frac{2 e^{-(2 n+1) \eta} \sin \phi}{\left(1-\nu^{2} \sin ^{2} \phi\right)(4 n+3)}\left[1+\frac{\rho}{4 n+3}+O\left(n^{-2}\right)\right]+O\left(e^{-4 n \eta}\right), \\
\rho & =1+\frac{\sin ^{2} \phi \sinh \eta}{\cosh \eta-1}\left[\frac{2\left(1-\nu^{2}\right)}{1-\nu^{2} \sin ^{2} \phi}-1\right]-\frac{\sinh \eta}{\cosh \eta-1}
\end{aligned}
$$

where $e^{\eta}$ is defined by (11) with $z=k^{\prime 2} \tan ^{2} \phi$. Then under the same conditions as in (29), with $\phi, k$ and $\nu$ fixed,

$$
\lim _{n \rightarrow \infty} S_{n}(\phi, k, \nu)=0 .
$$

The approximation (30) is convenient even when $k^{\prime 2} \tan ^{2} \phi>1$. Notice that if $\phi \rightarrow \pi / 2$ and $k \rightarrow 1$ so that $\cos ^{2} \phi=k^{\prime 2}$, then $k^{\prime 2} \tan ^{2} \phi \rightarrow 1$ and $e^{-\eta} \rightarrow 3-2^{3 / 2}$ $\approx 0.172$. The coefficient of $J\left(\phi, 1-\nu^{2}\right)$ in (30) can be replaced by

$$
-\nu^{2}\left[\left(1-\nu^{2}\right)\left(k^{2}-\nu^{2}\right)\right]^{-1 / 2}+\nu^{2}\left(1-\nu^{2}\right)^{-1} V_{n}\left(k^{\prime 2} /\left(1-\nu^{2}\right)\right)
$$

provided $\left|\arg \left(1-k^{\prime 2} /\left(1-\nu^{2}\right)\right)\right|<\pi$. However, this should not be introduced unless the magnitude of $V_{n}\left(k^{\prime 2} /\left(1-\nu^{2}\right)\right)$ is approximately equal to or less than the magnitude of $S_{n}(\phi, k, \nu)$.

To get a useful approximation when $\phi$ is near $\pi / 2$, we write 


$$
\begin{aligned}
F\left(\frac{\pi}{2}, k, \nu\right)-F(\phi, k, \nu) & =\int_{0}^{(\pi / 2)-\phi} \frac{\sec \alpha d \alpha}{\left(1-\nu^{2} \cos ^{2} \alpha\right)\left(k^{\prime 2}+\tan ^{2} \alpha\right)^{1 / 2}} \\
& =\int_{0}^{\psi} \frac{\sec \theta\left(1+{k^{\prime}}^{2} \tan ^{2} \theta\right)^{1 / 2} d \theta}{1-\nu^{2}+{k^{\prime}}^{2} \tan ^{2} \theta}, \\
k^{\prime} \tan \psi & =\cot \phi, \nu^{2} \sin ^{2} \phi \neq 1, \quad\left|\arg \left(1+{k^{\prime}}^{2} \tan ^{2} \psi\right)\right|<\pi .
\end{aligned}
$$

Now use (10). We find that

$$
\begin{aligned}
F\left(\frac{\pi}{2}, k, \nu\right)-F(\phi, k, \nu)= & \left(1-\nu^{2}\right)^{-1} J\left(\psi, \frac{k^{\prime 2}}{\left(1-\nu^{2}\right)}\right) \\
& \times\left[1-\frac{2\left(1-\nu^{2}\right)}{2 n+1} \sum_{m=1}^{n} \frac{\sin ^{2} \theta_{m}}{1-\left(1-\nu^{2}\right) \cos ^{2} \theta_{m}}\right] \\
& +\frac{2}{2 n+1} \sum_{m=1}^{n} \frac{\sin ^{2} \theta_{m}}{1-\left(1-\nu^{2}\right) \cos ^{2} \theta_{m}} \\
& \times J\left(\psi, k^{\prime 2} \cos ^{2} \theta_{m}\right)+M_{n}(\phi, k, \nu), \\
M_{n}(\phi, k, \nu)= & \int_{0}^{\psi} \frac{\sec \theta S_{n}\left(k^{\prime 2} \tan ^{2} \theta\right) d \theta}{1-\nu^{2}+k^{\prime 2} \tan ^{2} \theta} . \\
M_{n}(\phi, k, \nu)= & \frac{2\left(\cos \phi /\left(1+\sin ^{2} \phi\right)\right)^{4 n+1}(1-\sin \phi)}{\left(1-k^{2} \sin ^{2} \phi\right)^{1 / 2}\left(1-\nu^{2} \sin ^{2} \phi\right)(4 n+1)} \\
& \times\left[1+\frac{\sigma}{4 n+1}+O\left(n^{-2}\right)\right]+O\left(\frac{\cos \phi}{1+\sin ^{2}}\right)^{8 n} \\
\sigma= & 2 \sin \phi-1-\frac{k^{\prime 2} \sin ^{2} \phi}{1-k^{2} \sin ^{2} \phi}-\frac{2\left(1-\nu^{2}\right) \sin \phi}{1-\nu^{2} \sin ^{2} \phi}
\end{aligned}
$$

In (34), the coefficient of $J\left(\psi, k^{\prime 2} /\left(1-\nu^{2}\right)\right)$ can be replaced by $\nu\left(1-\nu^{2}\right)^{-1}-$ $\left(1-\nu^{2}\right)^{-1} W_{n}\left(1-\nu^{2}\right)$ provided $|\arg \nu|<\pi / 2$. This replacement should not be used unless approximately the magnitude of $W_{n}\left(1-\nu^{2}\right)$ is less than the magnitude of $M_{n}(\phi, k, \nu)$. If $\nu$ and $\phi$ are real, $e^{-\zeta}=(1-\nu) /(1+\nu)$ when $z=1-\nu^{2}$. Thus, use the alternative form provided

$$
(1-\nu) /(1+\nu) \leqq(1-\sin \phi) /(1+\sin \phi),
$$

i.e., when $\sin \phi \leqq \nu<1$. Under the same restrictions as in (33), with $\phi, k$, and $\nu$ fixed,

$$
\lim _{n \rightarrow \infty} M_{n}(\phi, k, \nu)=0 .
$$

Our technique is applicable to derive an expansion relating $K(k)=F(\pi / 2, k, 0)$ and $K\left(k^{\prime}\right)$, where $k^{\prime 2}=1-k^{2}$, since it can be shown that

$$
K(k)+\pi^{-1}\left(\ln {k^{\prime}}^{2}\right) K\left(k^{\prime}\right)=-\frac{2}{\pi} \int_{0}^{\pi / 2} \frac{\left(\ln \sin ^{2} \theta\right) d \theta}{\left(1-k^{\prime 2} \sin ^{2} \theta\right)^{1 / 2}} .
$$


Thus

$K(k)+\pi^{-1}\left(\ln {k^{\prime}}^{2}\right) K\left(k^{\prime}\right)=2(2 n+1)^{-1}\left[\ln 2+2 \sum_{m=1}^{n}{a_{m}}^{-1} \ln \left(1+a_{m}\right)\right]+A_{n}(k)$,

$$
\begin{gathered}
a_{m}=\left(1-n^{2} \sin ^{2} \theta_{m}\right)^{1 / 2} \\
A_{n}(k)=-\frac{2}{\pi} \int_{0}^{\pi / 2}\left(\ln \sin ^{2} \theta\right) V_{n}\left(k^{\prime 2} \sin ^{2} \theta\right) d \theta \\
A_{n}(k)=\left(\frac{k}{2 n \pi}\right)^{1 / 2}(2 n)^{-1}\left(\frac{1-k}{1+k}\right)^{2 n+1}\left[1-\frac{3\left(3 k^{2}+8 k-1\right)}{32 n k}+O\left(n^{-2}\right)\right] \\
+O\left(\left[\frac{1-k}{1+k}\right]^{4 n}\right) .
\end{gathered}
$$

Hence with $k$ fixed, $|\arg k|<\pi / 2$,

$$
\lim _{n \rightarrow \infty} A_{n}(k)=0 \text {. }
$$

\section{Approximations for the Elliptic Integral of the Second Kind. Let}

$$
\begin{aligned}
E(\phi, k)=\int_{0}^{\phi}\left(1-k^{2} \sin ^{2} \alpha\right)^{1 / 2} d \alpha & \\
& \left|\arg \left(1-k^{2}\right)\right|<\pi, \quad\left|\arg \left(1-k^{2} \sin ^{2} \phi\right)\right|<\pi .
\end{aligned}
$$

Here we use (2) and (15) - (19). Thus

$$
\begin{aligned}
E(\phi, k)= & (2 n+1) \phi-2(2 n+1)^{-1} \sum_{m=1}^{n} \tan ^{2} \theta_{m} H\left(\phi, k^{2} \cos ^{2} \theta_{m}\right)+T_{n}(\phi, k), \\
T_{n}(\phi, k)= & \int_{0}^{\phi} W_{n}\left(k^{2} \sin ^{2} \alpha\right) d \alpha . \\
T_{n}(\phi, k)= & -\frac{2 e^{-(2 n+1) \zeta} \tan \phi\left(1-k^{2} \sin ^{2} \phi\right)}{(4 n+1)}\left[1+\frac{\sigma_{1}}{4 n+1}+O\left(n^{-2}\right)\right] \\
& +O\left(e^{-4 n \zeta}\right), \\
\sigma_{1}= & \frac{2 k^{2} \sin ^{2} \phi(\cosh \zeta+1)}{\sinh \zeta}-\frac{(\cosh \zeta-1)}{\cos ^{2} \phi \sinh \zeta}-1,
\end{aligned}
$$

$$
\begin{aligned}
T_{n}\left(\frac{\pi}{2}, k\right)= & -\left[\frac{2 \pi(\cosh \zeta-1)}{(4 n+1) \sinh \zeta}\right]^{1 / 2} e^{-(2 n+1) \zeta}\left(1-k^{2} \sin ^{2} \phi\right) \\
\times & {\left[1+\frac{\sigma_{2}}{4 n+1}+O\left(n^{-2}\right)\right]+O\left(e^{-4 n \zeta}\right) } \\
\sigma_{2}= & \frac{\left(9 k^{2} \sin ^{2} \phi-2\right)(\cosh \zeta-1)}{8 \sinh \zeta}-\frac{1}{2}
\end{aligned}
$$


where $e^{\zeta}$ is defined by (5) with $z=k^{2} \sin ^{2} \phi$. Under the same conditions as for (41) with $\phi$ and $k$ fixed

$$
\lim _{n \rightarrow \infty} T_{n}(\phi, k)=0 \text {. }
$$

Numerical examples illustrating (42) and the remarkable efficiency of the error formulas for $T_{n}(\phi, k)$ are given in [1], [2].

After the manner of getting (30), we find

$$
\begin{aligned}
E(\phi, k)= & p_{n}(k) \sin \phi+\frac{2 k^{\prime 2}}{2 n+1} \sum_{m=1}^{n} \frac{\sin ^{2} \theta_{m} J\left(\phi, k^{\prime 2} \cos ^{2} \theta_{m}\right)}{1-k^{\prime 2} \cos ^{2} \theta_{m}} \\
& +U_{n}(\phi, k),\left|\arg \left(1+{k^{\prime}}^{2} \tan ^{2} \phi\right)\right|<\pi, \\
p_{n}(k)= & 1-\frac{2{k^{\prime}}^{2}}{2 n+1} \sum_{m=1}^{n} \frac{\sin ^{2} \theta_{m}}{1-{k^{\prime}}^{2} \cos ^{2} \theta_{m}}=k+\frac{2 k u}{1-u}, \quad u=\left(\frac{1-k}{1+k}\right)^{2 n+1}, \\
U_{n}(\phi, k)= & \int_{0}^{\phi} \cos \alpha S_{n}\left(k^{\prime 2} \tan ^{2} \alpha\right) d \alpha . \\
U_{n}(\phi, k)= & \frac{2 e^{-(2 n+1) \eta}\left(1-k^{2} \sin ^{2} \phi\right) \sin \phi}{(4 n+1)}\left[1+\frac{\mu}{4 n+1}+O\left(n^{-2}\right)\right]+O\left(e^{-4 n \eta}\right), \\
\mu= & \frac{2 \sinh n}{\cosh \eta+1}-1-\frac{3(\cosh \eta+1) \cos ^{2} \phi}{\sinh \eta}
\end{aligned}
$$

where $e^{\eta}$ is defined by (11) with $z=k^{\prime 2} \tan ^{2} \phi$. Thus for $\phi$ and $k$ fixed and restricted as in (45),

$$
\lim _{n \rightarrow \infty} U_{n}(\phi, k)=0 .
$$

In (45), we can take $p_{n}(k)=k$ provided that $|2 k u /(1-u)|<\left|U_{n}(\phi, k)\right|$. In this connection we notice that if $k$ and $\phi$ are real, then $(1-k) /(1+k)<e^{-\eta}$ when $k \tan \phi>1$.

A straightforward analysis shows that

$$
E(k)-E(\phi, k)=k^{\prime 2} F(\omega, k, k), \quad k^{\prime} \tan \omega=\cot \phi,
$$

and so we can apply the expansions previously developed as appropriate.

A relation analogous to (37) for the complete elliptic integral of the second kind is

$$
\begin{aligned}
E(k)= & \frac{2}{\pi} E\left(k^{\prime}\right)+\frac{2{k^{\prime}}^{2}}{\pi} K\left(k^{\prime}\right)-\frac{k^{\prime 2}}{\pi}\left\{2+\ln {k^{\prime}}^{2}\right\} \int_{0}^{\pi / 2} \frac{\sin ^{2} \theta d \theta}{\left(1-{k^{\prime}}^{2} \sin ^{2} \theta\right)^{1 / 2}} \\
& -\frac{2{k^{\prime}}^{2}}{\pi} \int_{0}^{\pi / 2} \frac{\sin ^{2} \theta\left(\ln \sin ^{2} \theta\right) d \theta}{\left(1-{k^{\prime}}^{2} \sin ^{2} \theta\right)^{1 / 2}},
\end{aligned}
$$

and from this one can derive the analogs of $(38)-(40)$. The result would be rather complicated and not as efficient as the known expansion for $E(k)$ in powers of $k^{\prime 2}$. Rather than use the expansion which could be found from (49), a much more efficient procedure would be to employ the Legendre relation, 


$$
E(k) K\left(k^{\prime}\right)+K(k) E\left(k^{\prime}\right)-K(k) K\left(k^{\prime}\right)=\pi / 2,
$$

and the appropriate expansions for $K(k)$ and $K\left(k^{\prime}\right)$.

Midwest Research Institute

Kansas City, Missouri 64110

1. Yudfll L. LukE, "Approximations for elliptic integrals," Math. Comp., v. 22, 1968, pp. 627-634. MR 37 \#2412.

2. YUdELL L. LUKE, The Special Functions and Their Approximations, Vol. 2, Academic Press, New York, 1969, pp. 269-273.

3. H. VAN DE VEL, "On the series expansion method for computing elliptic integrals of the first and second kinds," Math Comp., v. 23, 1969, pp. 61-70. 